\title{
People living with HIV easily lose their immune response to SARS-CoV-2: result from a cohort of COVID-19 cases in Wuhan, China
}

Yanbin Liu ${ }^{1 \dagger}$, Yanling Xiao ${ }^{1 \dagger}$, Songjie Wu ${ }^{2 \dagger}$, Gifty Marley ${ }^{3 \dagger}$, Fangzhao Ming ${ }^{4}$, Xiaoya Wang ${ }^{5}$, Mengmeng Wu ${ }^{1}$, Ling Feng ${ }^{1}$, Weiming Tang ${ }^{6,7^{*}+}$ and Ke Liang ${ }^{1,2,8,7^{*}+}$

\begin{abstract}
Background: To date, whether the immune response for SARS-CoV-2 infection among people living with HIV(PLWH) is different from HIV-naive individuals is still not clear.

Methods: In this cohort study, COVID-19 patients admitted to hospitals in Wuhan between January 15 and April 1, 2020, were enrolled. Patients were categorized into PLWH and HIV-naïve group. All patients were followed up regularly (every 15 days) until November 30, 2020, and the immune response towards SARS-CoV-2 was observed.

Results: Totally, 18 PLWH and 185 HIV-naïve individuals with COVID-19 were enrolled. The positive conversion rates of $\mathrm{lgG}$ were $56 \%$ in PLWH and 88\% in HIV-naïve patients respectively, and the peak was on the 45th day after COVID19 onset. However, the positive rate of IgG dropped to 12\% in PLWH and 33\% among HIV-naive individuals by the end of the study. The positive conversion rate of $\mathrm{lgG}$ among asymptomatic carriers is significantly lower than that among patients with moderate disease $(A O R=0.24,95 \% \mathrm{Cl} 0.07-0.85)$. PLWH had a lower lgG seroconversion rate ( $A O R=0.11,95 \% \mathrm{Cl} 0.03-0.39$ ) and shorter $\lg \mathrm{G}$ duration ( $\mathrm{AHR}=3.99,95 \% \mathrm{Cl} 1.43-11.13$ ) compared to HIV-naïve individuals. Patients with higher lymphocyte counts at onset had a lower positive conversion rate $(\mathrm{AOR}=0.30,95 \% \mathrm{Cl}$ 0.10-0.87) and shorter duration for $\operatorname{lgG}(\mathrm{AHR}=4.01,95 \% \mathrm{Cl} 1.78-9.02)$.
\end{abstract}

Conclusions: The positive conversion rate of lgG for SARS-CoV-2 was relatively lower and quickly lost in PLWH.

Keywords: SARS-CoV-2, COVID-19, People living with HIV (PLWH), Immune response

*Correspondence: Weiming_tang@med.unc.edu; keliang@whu.edu.cn ${ }^{\dagger}$ Yanbin Liu, Yanling Xiao, Songjie Wu, Gifty Marley, Weiming Tang and Ke Liang contributed equally to this work

${ }^{1}$ Department of Infectious Diseases, Zhongnan Hospital of Wuhan University, Wuhan, Hubei, China

${ }^{6}$ Dermatology Hospital of Southern Medical University, Guangzhou, China

Full list of author information is available at the end of the article

\section{Background}

The 2019 coronavirus disease (COVID-19) which is knowingly caused by the severe acute respiratory syndrome coronavirus 2 (SARS-CoV-2) has a strong global impact in the year 2020, and its impact is still ongoing [1]. However, to date, our comprehensive understanding of immune response for SARS-CoV-2 infection is still questionable as clinical findings continue to contradict each other [2-4].

For example, a study in Iceland concluded that antibodies for SARS-CoV-2 did not decline within 
4 months after diagnosis [2]. In direct contrast, other comparative studies invariably observed a substantial decrease in antibodies overtime after infection $[3,4]$, the last study in Wuhan also revealed the antibodies significantly decreased in 6 months after the acute phase [5]. Moreover, specific antibodies in mild patients were undoubtedly found to disappear more rapidly [6]. In addition, empirical findings from some studies showed that SARS-CoV-2-specific antibodies could offer protection against reinfection by providing the rationale for the administration of plasma containing SARS-CoV-2 neutralizing antibodies as a treatment for COVID-19 [7, 8]. However, some case studies have also reported that people who recovered from COVID19 can still be re-infected with SARS-CoV-2 in a relatively short time $[9,10]$. This raised global concerns regarding how long the specific antibodies can last and function effectively within the body post-SARS-CoV-2 infection [11].

For people living with $\operatorname{HIV}(\mathrm{PLWH})$ infected with SARS-CoV-2, the clinical conditions may be more complicated for their immunodeficiency and immune dysregulation [12]. Published studies from Spain and our former study in Wuhan both showed that COVID-19 in PLWH might be more severe [13, 14]. But some current study findings tentatively suggest no difference in the incidence rate and adverse outcomes of COVID-19 between PLWH and the other individuals $[15,16]$. A recent study proposed people with HIV in the UK seem to be at increased risk of COVID-19 mortality [17], but other researchers were skeptical about this statement [18]. In addition, there is very limited information on whether the immune response to SARS-CoV-2 infection is similar in PLWH and HIV-naïve individuals.

To fill this gap, we conducted a cohort study among both HIV infected and HIV-naïve COVID-19 patients in Wuhan, China, to understand the immune response among these individuals.

\section{Methods}

\section{Study design and participants' recruitment}

COVID-19 patients (age $>18$ years) who were hospitalized in the Department of Infectious Diseases of Wuhan University Zhongnan Hospital and Wuhan NO.7 Hospital between January 15 and April 1, 2020 were recruited. Among all 248 inpatients age $>18$ years, 203 were enrolled in this study. Patients were categorized into groups with HIV and without HIV. The diagnosis and classification of disease severity were defined based on the "New Coronavirus Pneumonia Prevention and Control Program (8th edition)" published by the National Health Commission of China [19].

\section{Laboratory procedures}

Nucleic acid tests (NAT) for SARS-CoV-2 were conducted using real-time reverse transcriptional polymerase chain reaction (RT-PCR) kits as recommended by the Chinese center for disease control and prevention (CDC) following the WHO guidelines. Gold immunochromatography assay (qualitative test) was used in testing the IgG and IgM antibodies response against SARS-CoV-2 spike protein and nucleocapsid protein. All test kits used were approved by the China Food and Drug Administration and provided by Zhuhai Livzon Diagnostics Inc.

\section{Data collection and follow-up}

Participants' data were collected from January 15, 2020, including gender, age, comorbidities, smoking, lymphocyte counts when illness onset, COVID-19 severity, and the time of disease diagnosis. PLWH participants' data were acquired from the China CDCs' AIDS Comprehensive Prevention and Control Data Information Management System. Required data and key information for HIV-naïve patients were acquired from their electronic clinical records. Comorbidities included hypertension, heart disease, diabetes, chronic liver and kidney disease. All COVID-19 diagnosed patients were followed up regularly (every 15 days) until November 30, 2020. NAT and antibody tests were done at each follow-up.

\section{Statistical analysis}

Categorical variables were presented as counts (\%), and continuous variables were presented as median (interquartile range, IQR). Univariate and multivariable logistic regressions were used to identify factors associated with antibodies-positive rates. Odds ratios (OR) with 95\% confidence intervals $(\mathrm{CI})$ and P-values were reported. The Kaplan-Meier method was used to estimate the cumulative probability of IgG and IgM negative conversion and the median duration time of IgG and IgM. The Cox proportional hazards regression model was used to examine the factors associated with the duration time of IgG and IgM after controlling for confounders including gender, age, comorbidities, smoking, lymphocyte count when illness onset, COVID-19 severity, and HIV status. The adjusted hazard ratios (AHR) and 95\% CI were calculated in the model. Statistical significance was defined as a two-sided P-value of less than 0.05. All statistical analyses were conducted using SPSS26.0.

\section{Results}

\section{Characteristics of enrolled patients}

In total, 203 COVID-19 patients were enrolled in the study, including $18 \mathrm{PLWH}$ and 185 HIV-naïve individuals. The proportion of females in the HIV-naïve 
population was significantly higher than the PLWH group (62\% vs 6\%, P $<0.001$ ), and the PLWHs group had a higher proportion of asymptomatic infected patients compared to the HIV-naïve group ( $33 \%$ vs $6 \%, \mathrm{P}<0.001)$. The positive conversion rates of IgG were $56 \%$ and $88 \%$ in the PLWH and the HIV-naïve group, respectively $(\mathrm{P}=0.001)$ (Table 1). For PLWH, the CD4+ lymphocyte counts (CD4 count) was $452(12-612)$ cells $/ \mu \mathrm{L}, 72.2 \%$ $(13 / 18)$ had viral suppressed, $83.3 \%$ (15/18) were receiving antiretroviral therapy (ART).

\section{Antibody positive conversion rates and associated factors}

The positive conversion rate of IgG among asymptomatic carriers is $44 \%$, which is significantly lower than the observed rate of $88 \%$ among patients with moderate disease (adjusted odds ratio $(\mathrm{AOR})=0.24,95 \% \mathrm{CI}$ 0.07-0.85). We also found that although there was no statistically significant difference in IgM seroconversion between the two groups, PLWH group members had a lower IgG seroconversion rate compared to the HIV-naive group (AOR $=0.11,95 \%$ CI $0.03-0.39$ ). The conversion rate of IgG was decreased with the increase of lymphocyte counts (AOR $=0.30,95 \%$ CI $0.10-0.87$ ) (Table 2). The antibody-positive conversion rate reaches the peak at the 45th day after onset, then begins to decline (Fig. 1).
Table 2 The antibody positive conversion rates and associated factors among COVID-19 cases in Wuhan, China, 2020 ( $N=203)$

\begin{tabular}{lll}
\hline Variables & $\begin{array}{l}\text { IgM (AOR and 95\% } \\
\text { CI) }\end{array}$ & IgG (AOR and 95\% Cl) \\
\hline Type & & \\
Moderate & Ref & Ref \\
Asymptomatic & $0.35(0.09-1.40)$ & $0.24(0.07-0.85)$ \\
Mild & $0.75(0.06-8.92)$ & $2.75 \times 10^{8}(0.00-N A)$ \\
Severe & $2.28(0.73-7.20)$ & $0.58(0.13-2.63)$ \\
Co-infected with HIV & $0.58(0.18-1.82)$ & $0.11(0.03-0.39)$ \\
Lymphocyte counts & $0.48(0.22-1.08)$ & $0.30(0.10-0.87)$ \\
$\left(10^{9} /\right.$ L) & & \\
\hline
\end{tabular}

${ }^{a}$ Each association was mutually adjusted for the other characteristics in the table

\section{Cumulative duration of antibodies and associated factors}

The median duration time of IgG and IgM negative conversion since disease onset was 223 days (95\% CI 184-NA) and 112 days (95\% CI 107-116) respectively. The results of the Cox-proportional hazard regression model adjusted for confounders were shown in Table 3. With the increase of age, the duration time of IgM was increased (AHR $=0.96,95 \%$ CI 0.93-0.98). PLWH had a shorter IgG duration (AHR $=3.99,95 \%$ CI 1.43-11.13) and patients with higher lymphocyte counts at onset had a shorter IgG duration (AHR $=4.01,95 \%$ CI 1.78-9.02).

Table 1 Characteristics of enrolled patients with COVID-19, Wuhan, China, $2020(N=203)$

\begin{tabular}{|c|c|c|c|c|c|}
\hline & \multicolumn{2}{|c|}{ HIV negative $(n=185)$} & \multicolumn{2}{|c|}{ HIV positive $(n=18)$} & \multirow[t]{2}{*}{ P value } \\
\hline & $\mathbf{n}$ & $\%$ & $\mathbf{n}$ & $\%$ & \\
\hline Age, years & $45(34-57)$ & & $43(35-50)$ & & 0.75 \\
\hline \multicolumn{6}{|l|}{ Gender } \\
\hline Male & 71 & 38.38 & 17 & 94.44 & $<0.001$ \\
\hline Female & 114 & 61.62 & 1 & 5.56 & \\
\hline \multicolumn{6}{|l|}{ Comorbidities $^{\mathrm{a}}$} \\
\hline No & 156 & 84.32 & 12 & 66.67 & 0.09 \\
\hline Yes & 29 & 15.68 & 6 & 33.33 & \\
\hline \multicolumn{6}{|l|}{ Smoking } \\
\hline No & 149 & 80.54 & 17 & 94.44 & 0.21 \\
\hline Yes & 36 & 19.46 & 1 & 5.56 & \\
\hline Lymphocyte counts $\left(10^{9} / \mathrm{L}\right)$ & $1.00(0.36-1.76)$ & & $1.60(1.22-1.79)$ & & 0.72 \\
\hline \multicolumn{6}{|l|}{ Types of COVID-19 } \\
\hline Asymptomatic carriers & 12 & 6.49 & 6 & 33.33 & $<0.001$ \\
\hline Mild & 4 & 2.16 & 0 & 0.00 & \\
\hline Moderate & 156 & 84.32 & 9 & 50.00 & \\
\hline Severe & 13 & 7.03 & 3 & 16.67 & \\
\hline Positive conversion of IgM & 96 & 51.89 & 6 & 33.33 & 0.15 \\
\hline Positive conversion of lgG & 163 & 88.11 & 10 & 55.56 & 0.001 \\
\hline
\end{tabular}

Data are presented as count (\%) or median (IQR)

${ }^{a}$ Including hypertension, heart disease, diabetes, chronic liver and kidney disease 


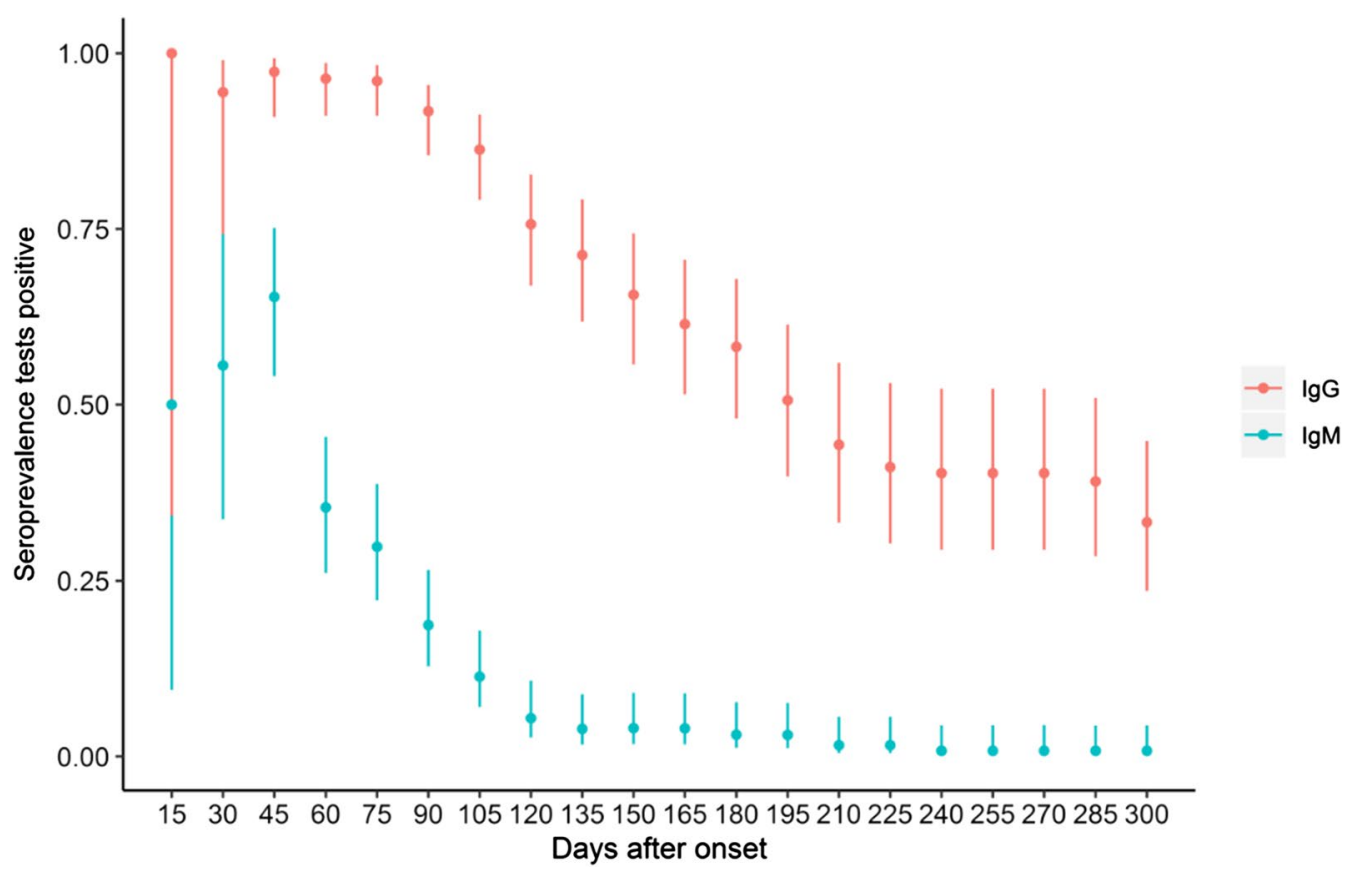

Fig. 1 The variation trend of antibody positive rates among COVID-19 cases in Wuhan, China, 2020. The range are expressed as 95\% confidence interval

Table 3 The associated factors of IgG and IgM duration time among COVID-19 cases in Wuhan, China, 2020 ( N=203)

\begin{tabular}{lll}
\hline Variables & $\begin{array}{l}\text { IgM (AHR and 95\% } \\
\text { CI) }\end{array}$ & IgG (AHR and 95\% Cl) \\
\hline Age & $0.96(0.93-0.98)$ & $1.25(0.53-2.91)$ \\
Gender & & \\
Male & Ref & Ref \\
Female & $1.47(0.71-3.04)$ & $0.99(0.96-1.00)$ \\
Current smoking & & \\
No & Ref & Ref \\
Yes & $0.69(0.19-2.58)$ & $0.83(0.09-7.24)$ \\
Comorbidities & & \\
No & Ref & Ref \\
Yes & $1.23(0.55-2.72)$ & $1.29(0.33-5.05)$ \\
COVID-19 severity & & \\
Moderate & Ref & Ref \\
Mild & $0.79(0.09-6.84)$ & $0.65(0.14-2.94)$ \\
Severe & $1.57(0.67-3.68)$ & $0.85(0.18-3.98)$ \\
Co-infected with HIV & & \\
No & Ref & Ref \\
Yes & $1.23(0.44-3.44)$ & $3.99(1.43-11.13)$ \\
Lymphocyte counts & $0.86(0.33-2.24)$ & $4.01(1.78-9.02)$ \\
(10 $\%$ L $)$ & & \\
\hline
\end{tabular}

a Each association was mutually adjusted for the other characteristics in the table

\section{Discussion}

Understanding the immune response towards SARSCoV-2 among both PLWH and uninfected individuals is essential to providing tailored prevention and treatment measures against COVID-19. Findings from this study extend current literature by evaluating the similarities and differences in the immune response to SARS-CoV-2 infection between PLWH and HIV-naïve patients $[3,4,6]$. Compared to the HIV-naïve group, we found a lower positive conversion rate of IgG in PLWH, and the antibodies were lost much quicker.

Our findings showed that IgG positive conversion rate among COVID-19 infected individuals is higher in HIV-naïve patients (88\%) than PLWH (56\%). Similar to our findings, a study conducted in Iceland obtained 91\% positive pan-immunoglobulin antibodies [2] and another in Chongqing of China reported an $84 \%$ IgG positive rate among HIV-naïve COVID-19 patients [3]. This observation is however not surprising as PLWH are likely to have imbalanced immune systems which could cause fewer antibody productions in the case of COVID-19 [20]. In addition, findings from other studies suggested that an impaired immune reactivity could contribute to low IgG positive [21]. Although this may suggest that PLWH may be more vulnerable with a SARS-CoV-2 infection, findings from other studies have observed no increased risk and severity of COVID-19 
in affected PLWH [22]. Regardless, the risk of increased PLWH vulnerability should not be ignored.

We further discovered that the duration time for IgG positive conversion is shorter in PLWH. According to our findings, the positive conversion rates of IgG were 56\% in PLWH and $88 \%$ in HIV-naïve patients respectively, and the peak was on the 45th day after COVID-19 onset. However, the positive rate of IgG dropped to $12 \%$ in PLWH and 33\% among HIV-naïve individuals by the last observation time. In addition, a Cox proportional hazards regression conducted showed the IgG duration time in PLWH to be shorter than the general population. In explanation, findings from some case reports had shown specific antibodies response to be delayed or even vanish in PLWH with compromised immune status due to CD4 count depletion and B lymphocytes dysfunction [23]. We, therefore, speculate that immune deficiency in PLWH could account for the low antibody response to SARS$\mathrm{CoV}-2$ infection and short cumulative duration time. The quick loss of antibodies may imply a higher susceptibility to reinfections as previous studies have demonstrated the presence of IgG antibodies to be essential in reducing the risks of reinfection in the ensuing 6 months post treatment [24]. Some literatures had shown neutralizing antibodies and special antibodies could collaborate to neutralize and eliminate the virus $[25,26]$, so our result also suggested PLWH had poorer capacity to eliminate the virus. Some studies have however stipulated that ART may provide some form of protection against COVID-19 incidence given the effect some antiretroviral drugs have on the SARS-CoV-2 life cycle [27]. So, to positively enhance active immunity, encouraging treatment adherence is both important and urgent.

We found that the severity of COVID-19 was associated with the positive conversation rate of IgG, and lymphocyte counts at illness onset had an effect on both positive conversion rate and duration time of IgG. Our findings invariably showed a lower positive conversion rate of IgG in asymptomatic carriers from each patient group. This finding concurs with findings from previous studies conducted in Korea and Wuhan, China [28, 29]. Some studies have attributed that low level of viral loads that lead to low antibody response in asymptomatic individuals may have accounted for these findings [30, 31]. The attribution is plausibility as high viral loads such as those found in severe patients trigger a stronger antibody response. Through increased levels of viral loads, there is a more significant depletion of lymphocyte [32] and a high positive conversion rate of antibodies in severe disease [33,34], thus, result in a longer IgG duration in patients with lower lymphocyte counts at onset.

Our study includes some limitations. First, because of the fundamental lack of an antibody detection kit in the early days of the SARS-CoV-2 epidemic in Wuhan, the possible opportunity for early antibody testing lacked. This however exerts no direct influence on our comparison of immune response between the two patient groups. Second, the specific number of COVID-19 infected PLWH was relatively small in our study. For this reason, our study was naturally limited in its power to detect differences among COVID-19 patients with different HIV statuses. Third, due to the limited information collected in this study, many of the potential confounders were not adjusted which may bias our results.

\section{Conclusion}

In conclusion, our study revealed that the positive conversion rate of the SARS-CoV-2 specific antibodies was relatively lower and quickly lost in PLWH with COVID-19.

\section{Abbreviations \\ COVID-19: 2019 Coronavirus disease; SARS-CoV-2: Severe acute respiratory syndrome coronavirus 2; PLWH: People living with HIV; CDC: The Chinese center for disease control and prevention; NAT: Nucleic acid tests; RT-PCR: Real-time reverse transcriptional polymerase chain reaction; IQR: Interquartile range; OR: Odds ratios; Cl: Confidence intervals; AHR: Adjusted hazard ratios; CD4 count: CD4+ lymphocyte counts; ART: Antiretroviral therapy.}

\section{Acknowledgements}

We would like to show our respect and gratitude to all the health workers who are at the first line of the outbreak response and fighting against COVID-19.

\section{Authors' contributions}

WT and KL conceived and designed this investigation. YL, FM and MW helped to design the scheme of the investigation. YX, FM, XW, and LF collected the original data. SW analyzed the data. SW, WT, GM, and KL contributed to the interpretation of the data. YL, MG, WT, GM, and KL contributed to the writing of the paper. All authors read and approved the final manuscript.

\section{Funding}

This work was supported by the National Nature Science Foundation of China (81903371), NIMH (R34MH1 19963), the National Science and Technology Major Project (2018ZX10101-001-001-003), and Special Found on Prevention and Control of New Coronary Pneumonia in Guangdong Universities (2020KZDZX1047), Medical Science and Technology Innovation Platform Support Project of Zhongnan Hospital, Wuhan University (PTXM2020008), Science and Technology Innovation Cultivation Fund of Zhongnan Hospital, Wuhan University (cxpy2017043), and the Medical Science Advancement Program (Basic Medical Sciences) of Wuhan University (TFJC2018004).

\section{Availability of data and materials}

The datasets used and/or analysed during the current study are available from the corresponding author on reasonable request.

\section{Declarations}

\section{Ethics approval and consent to participate}

This study was approved by the Ethics Committee of Zhongnan Hospital affiliated to Wuhan University (2020062) and followed the recommendations of Helsinki Declaration. All participants signed the informed consent form.

Consent for publication

Not applicable. 


\section{Competing interests}

The authors declare no competing interests.

\section{Author details}

${ }^{1}$ Department of Infectious Diseases, Zhongnan Hospital of Wuhan University, Wuhan, Hubei, China. ${ }^{2}$ Department of Nosocomial Infection Management, Zhongnan Hospital of Wuhan University, Hubei, China. ${ }^{3}$ School of Public Health, Nanjing Medical University, Nanjing, China. ${ }^{4}$ Wuchang District Center for Disease Control and Prevention, Wuhan, Hubei, China. ${ }^{5}$ Wuhan No.7 People's Hospital, Wuhan, Hubei, China. ${ }^{6}$ Dermatology Hospital of Southern Medical University, Guangzhou, China. ${ }^{7}$ The University of North Carolina at Chapel Hill Project-China, Guangzhou 510095, China. ${ }^{8}$ Wuhan Research Center for Infectious Diseases and Cancer, Chinese Academy of Medical Sciences, Wuhan, China. ${ }^{9}$ Center of Preventing Mother-to-Child Transmission for Infectious Diseases, Zhongnan Hospital of Wuhan University, Wuhan, China.

Received: 20 May 2021 Accepted: 9 September 2021

Published online: 01 October 2021

\section{References}

1. Huang C, Wang Y, Li X, Ren L, Zhao J, Hu Y, et al. Clinical features of patients infected with 2019 novel coronavirus in Wuhan, China. Lancet. 2020;395(10223):497-506. https://doi.org/10.1016/S0140-6736(20) 30183-5.

2. Gudbjartsson DF, Norddahl GL, Melsted P, Gunnarsdottir K, Holm H, Eythorsson E, et al. Humoral immune response to SARS-CoV-2 in Iceland. N Engl J Med. 2020;383(18):1724-34. https://doi.org/10.1056/NEJMo a2026116.

3. Long QA-O, Tang XJ, Shi QL, Li Q, Deng HA-OX, Yuan J, et al. Clinical and immunological assessment of asymptomatic SARS-CoV-2 infections. Nat Med. 2020;26(8):1200-4. https://doi.org/10.1038/s41591-020-0965-6.

4. Seow J, Graham C, Merrick B, Acors S, Pickering S, Steel KJA, et al. Longitudinal observation and decline of neutralizing antibody responses in the three months following SARS-CoV-2 infection in humans. Nat Microbiol. 2020;5(12):1598-607. https://doi.org/10.1038/s41564-020-00813-8.

5. Huang C, Huang L, Wang Y, Li X, Ren L, Gu X, et al. 6-month consequences of COVID-19 in patients discharged from hospital: a cohort study. The Lancet. 2021. https://doi.org/10.1016/S0140-6736(20)32656-8.

6. Ibarrondo FJ, Fulcher JA, Goodman-Meza D, Elliott J, Hofmann C, Hausner MA, et al. Rapid decay of anti-SARS-CoV-2 antibodies in persons with mild Covid-19. N Engl J Med. 2020;383(11):1085-7. https://doi.org/10.1056/ NEJMc2025179 (Epub 2020 Jul 21)

7. Sharun K, Tiwari R, Iqbal Yatoo M, Patel SK, Natesan S, Dhama J, et al. Antibody-based immunotherapeutics and use of convalescent plasma to counter COVID-19: advances and prospects. Expert Opin Biol Ther. 2020;20(9):1033-46. https://doi.org/10.1080/14712598.2020.1796963.

8. Chen L, Xiong J, Bao L, Shi Y. Convalescent plasma as a potential therapy for COVID-19. Lancet Infect Dis. 2020;20(4):398-400. https://doi.org/10. 1016/S1473-3099(20)30141-9.

9. Lafaie L, Célarier T, Goethals LA-O, Pozzetto B, Grange S, Ojardias EA-O, et al. Recurrence or relapse of COVID-19 in older patients: a description of three cases. J Am Geriatr Soc. 2020;68(10):2179-83. https://doi.org/10. $1111 /$ jgs. 16728 .

10. Tillett RL, Sevinsky JR, Hartley PD, Kerwin H, Crawford N, Gorzalski A, et al. Genomic evidence for reinfection with SARS-CoV-2: a case study. Lancet Infect Dis. 2020;21(1):52-8. https://doi.org/10.1016/S1473-3099(20) 30764-7.

11. White LV, Lee N, Marin FP, Saludar NR, Edwards T, Cox SE, et al. Performance of alternative measures to body mass index in the assessment of moderate and severe under-nutrition among acutely unwell patients hospitalized in a TB ward in the Philippines: a cross-sectional study. PLoS ONE. 2019;14(5):e0215968. https://doi.org/10.1371/journal.pone.0215968.

12. Vizcarra P, Pérez-Elías MJ, Quereda C, Moreno A, Vivancos MJ, Dronda F, et al. Description of COVID-19 in HIV-infected individuals: a single-centre, prospective cohort. Lancet HIV. 2020;7(8):e554-64. https://doi.org/10. 1016/\$2352-3018(20)30164-8.

13. Del Amo J, Polo R, Moreno SA-O, Díaz AA-O, Martínez E, Arribas JA-O, et al. Incidence and severity of COVID-19 in HIV-positive persons receiving antiretroviral therapy : a cohort study. Ann Intern Med. 2020;173(7):536-41. https://doi.org/10.7326/M20-3689.

14. Guo WA-O, Ming F, Feng Y, Zhang Q, Mo P, Liu L, et al. Patterns of HIV and SARS-CoV-2 co-infection in Wuhan, China. (1758-2652 (Electronic)).

15. British HIV Association. BHIVA, DAIG, EACS, GESIDA \& Polish Scientific AIDS Society Statement on risk of COVID-19 for people living with HIV (PLWH) 2020. Accessed 24 Oct 2020. https://www.bhiva.org/BHIVA-DAIG-EACSGESIDA-Polish-Scientific-AIDS-Society-statement-on-risk-of-COVID-19for-PLWH. Accessed 24 Oct 2020.

16. Sigel K, Swartz T, Golden E, Paranjpe I, Somani S, Richter F, et al. Covid-19 and people with HIV infection: outcomes for hospitalized patients in New York City. Clin Infect Dis. 2020;71(11):2933-8. https://doi.org/10.1093/cid/ ciaa880.

17. Bhaskaran K, Rentsch CT, MacKenna B, Schultze A, Mehrkar A, Bates CJ, et al. HIV infection and COVID-19 death: a population-based cohort analysis of UK primary care data and linked national death registrations within the OpenSAFELY platform. Lancet HIV. 2020;8(1):e24-32. https:// doi.org/10.1016/S2352-3018(20)30305-2 (Epub 2020 Dec 11).

18. Waters LJ, Pozniak AL. COVID-19 death in people with HIV: interpret cautiously. Lancet HIV. 2020;8(1):e2-3. https://doi.org/10.1016/S23523018(20)30332-5

19. National Health Commission. Diagnosis and treatment of COVID-19 pneumonia in China (the 8th edition). 2020. http://www.nhc.gov.cn/ yzygj/s7653p/202008/0a7bdf12bd4b46e5bd28ca7f9a7f5e5a.shtml.

20. Gervasoni C, Meraviglia P, Riva A, Giacomelli A, Oreni L, Minisci D, et al. Clinical features and outcomes of HIV patients with coronavirus disease 2019. Clin Infect Dis. 2020;71(16):2276-8. https://doi.org/10.1093/cid/ ciaa579.

21. Hou H, Wang T, Zhang B, Luo Y, Mao L, Wang F, et al. Detection of IgM and IgG antibodies in patients with coronavirus disease 2019. Clin Transl Immunol. 2020:9(5):e01136. https://doi.org/10.1002/cti2.1136.

22. Mondi A, Cimini E, Colavita F, Cicalini S, Pinnetti C, Matusali G, et al. COVID-19 in people living with HIV: clinical implications of dynamics of the immune response to SARS-CoV-2. J Med Virol. 2020. https://doi.org/ 10.1002/jmv.26556 (Electronic).

23. Wang M, Luo L, Bu H, Xia H. One case of coronavirus disease 2019 (COVID-19) in a patient co-infected by HIV with a low CD4(+) T-cell count. Int J Infect Dis. 2020;96:148-50. https://doi.org/10.1016/j.ijid.2020. 04.060 .

24. Lumley SF, O'Donnell D, Stoesser N, Matthews PC, Howarth A, Hatch SB, et al. Antibody status and incidence of SARS-CoV-2 infection in health care workers. N Engl J Med. 2020. https://doi.org/10.1056/NEJMoa2034 545.

25. Lagunas-Rangel FA, Chávez-Valencia V. What do we know about the antibody responses to SARS-CoV-2? Immunobiology. 2021;226(2):152054. https://doi.org/10.1016/j.imbio.2021.152054.

26. Salazar E, Christensen PA, Graviss EA, Nguyen DT, Castillo B, Chen J, et al. Significantly decreased mortality in a large cohort of Coronavirus Disease 2019 (COVID-19) patients transfused early with convalescent plasma containing high-titer anti-severe acute respiratory syndrome coronavirus 2 (SARS-CoV-2) spike protein IgG. Am J Pathol. 2021;191(1):90-107. https:// doi.org/10.1016/j.ajpath.2020.10.008.

27. Patel R, Acharya A, Mohan M, Byrareddy S. COVID-19 and AIDS: outcomes from the coexistence of two global pandemics and the importance of chronic antiretroviral therapy. J Med Virol. 2020;93(2):641-3. https://doi. org/10.1002/jmv.26416.

28. Ko JH, Joo EJ, Park SJ, Baek J, Kim WD, Jee J, et al. Neutralizing antibody production in asymptomatic and mild COVID-19 Patients, in comparison with pneumonic COVID-19 patients. J Clin Med. 2020;9(7):2268. https:// doi.org/10.3390/jcm9072268.

29. Lei Q, Li Y, Hou HY, Wang F, Ouyang ZQ, Zhang Y, et al. Antibody dynamics to SARS-CoV-2 in asymptomatic COVID-19 infections. Allergy. 2020. https://doi.org/10.1111/all.14622.

30. Shields A, Faustini SE, Perez-Toledo M, Jossi S, Aldera E, Allen JD, et al. SARS-CoV-2 seroprevalence and asymptomatic viral carriage in healthcare workers: a cross-sectional study. Thorax. 2020;75(12):1089-94. https://doi.org/10.1136/thoraxjnl-2020-215414.

31. Yongchen Z, Shen H, Wang X, Shi X, Li Y, Yan J, et al. Different longitudinal patterns of nucleic acid and serology testing results based on disease severity of COVID-19 patients. Emerg Microbes Infect. 2020;9(1):833-6. https://doi.org/10.1080/22221751.2020.1756699. 
32. Chen W, Xiao Q, Fang Z, Lv X, Yao M, Deng M. Correlation analysis between the viral load and the progression of COVID-19. Comput Math Methods Med. 2021;2021:9926249. https://doi.org/10.1155/2021/99262 49.

33. Guan W, Ni ZY, Hu Y, Liang WH, Ou CQ, He JX, et al. Clinical characteristics of coronavirus disease 2019 in China. N Engl J Med. 2020;382(18):170820. https://doi.org/10.1056/NEJMoa2002032.

34. Tan L, Wang Q, Zhang D, Ding J, Huang Q, Tang YQ, et al. Lymphopenia predicts disease severity of COVID-19: a descriptive and predictive study.
Signal Transduct Target Ther. 2020;5(1):33. https://doi.org/10.1038/ s41392-020-0148-4

\section{Publisher's Note}

Springer Nature remains neutral with regard to jurisdictional claims in published maps and institutional affiliations.
Ready to submit your research? Choose BMC and benefit from:

- fast, convenient online submission

- thorough peer review by experienced researchers in your field

- rapid publication on acceptance

- support for research data, including large and complex data types

- gold Open Access which fosters wider collaboration and increased citations

- maximum visibility for your research: over $100 \mathrm{M}$ website views per year

At BMC, research is always in progress.

Learn more biomedcentral.com/submissions 